Musées, Patrimoine et Culture scientifiques et techniques

$136 \mid 2011$

juillet - aôut 2011

\title{
Dix ans d'acquisitions au muséum de Bordeaux
}

Ten years of acquiring collection objects at Bordeaux's Museum

\section{Nathalie Mémoire}

URL : http://journals.openedition.org/ocim/884

DOI : 10.4000/ocim.884

ISSN : 2108-646X

Éditeur

OCIM

Édition imprimée

Date de publication : 1 juillet 2011

Pagination : 5-13

ISSN : 0994-1908

Référence électronique

Nathalie Mémoire, «Dix ans d'acquisitions au muséum de Bordeaux », La Lettre de l'OCIM [En ligne],

136 | 2011, mis en ligne le 01 juillet 2013, consulté le 04 mai 2019. URL : http://

journals.openedition.org/ocim/884; DOI : 10.4000/ocim.884 


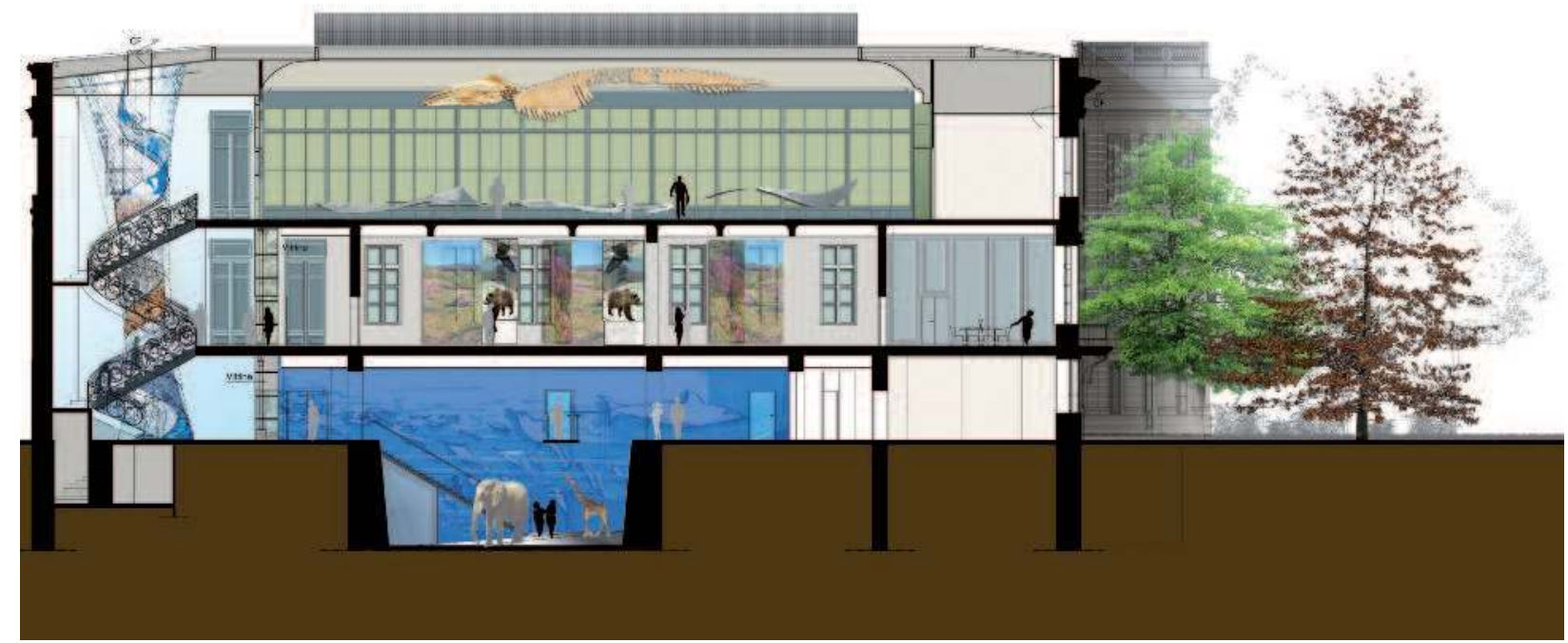

Esquisse du projet architectural du futur muséum de Bordeaux : les points forts portent sur le traitement de la galerie XIXe siècle, dans le respect de l'architecture d'origine, la création d'un espace d'exposition temporaire en sous-sol, ainsi qu'une entrée et un espace d'accueil créant une transition harmonieuse entre le parc et les espaces intérieurs grâce à une pente douce venant se glisser sous le bâtiment. (c) Muséum de Bordeaux

dans une mise en scène modulable, des présentations dites semi-permanentes qui permettront de faire tourner le fonds et des expositions temporaires créant l'événement.

L'affectation au muséum de Bordeaux d'un premier local de réserves, inauguré en septembre 2001, a rapidement permis d'envisager le stockage de collections non exposées en permanence mais aussi l'entrée de nouveaux spécimens qui pourraient soit illustrer ces futurs parcours, soit enrichir les collections parfois à l'occasion des expositions temporaires programmées, soit pour développer ces collections d'étude destinées à répondre aux demandes de professionnels ou d'étudiants, le tout dans le cadre du projet scientifique et culturel de l'établissement développé à l'occasion du projet de rénovation.

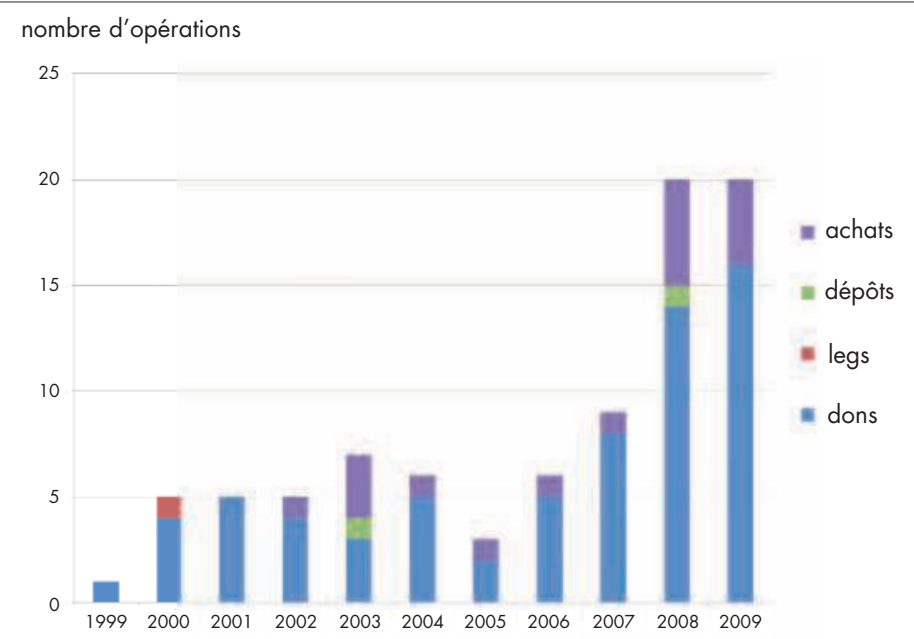

Les opérations d'acquisitions entre 1999 et 2009
Au nombre d'une centaine d'entrées en dix ans, ces acquisitions correspondent à un effectif variant de un à quelques centaines, parfois milliers de spécimens ou échantillons de nature et de tailles très diverses, diverses aussi par leur mode d'acquisition (legs, dons, achats, dépôts) et leur statut (patrimonial, pédagogique, d'étude, ou transitoire comme les dépouilles «en attente de naturalisation ») ; au total environ 18000 spécimens. Les achats ont été financés par la Ville de Bordeaux, à plusieurs reprises avec l'aide du FRAM (Fonds régional d'acquisition pour les musées).

\section{Spécificité des collections d'Histoire naturelle}

Les collections du patrimoine naturel et scientifique ne sont pas des œuvres d'art. Elles n'ont pas pour vocation de témoigner du génie humain.

Chronologiquement, elles ont été réunies d'abord pour montrer les curiosités de la Nature, puis étudier et tenter de comprendre la diversité et l'organisation de celle-ci, enfin pour expliciter cette organisation et sa genèse. Aujourd'hui, elles sont utilisées pour mettre en évidence, auprès du plus large public, les interrelations et les équilibres fragiles dont nous prenons une conscience tardive. Ainsi, ces collections sont composées pour partie de vestiges naturels, minéraux ou organiques, bruts de collecte, ou au contraire mis en forme de manière à reconstituer un animal représentatif d'une espèce, une scène représentative d'une situation ou d'un environnement particulier. Il s'agit ainsi d'éléments en partie naturels et en partie reconstitués afin de servir de support à l'exposé d'un discours scientifique.

\section{Pourquoi continuer à acquérir ?}

Les collections composant le riche fonds patrimonial du muséum d'Histoire naturelle de Bordeaux ont été réunies 
principalement entre la fin du XVIIIe siècle et le tout début des années 1960. Jusqu'à une période très récente (moins de quinze ans), les préoccupations concernant la conservation préventive dans les musées n'avaient pas complètement émergé. Si des traitements chimiques contre les insectes ravageurs des matières organiques (peaux, plumes et poils) ont été très tôt mis en œuvre par nos prédécesseurs, les autres causes de détériorations n'étaient pas identifiées. Là comme ailleurs, les collections ont été exposées à la lumière du jour, conditions qui ont entraîné, pour certaines d'entre elles, d'importantes décolorations ; elles ont aussi subi d'importantes variations de température et d'hygrométrie qui ont parfois entraîné des déchirures.

Pour leur présentation en exposition temporaire ou dans le futur parcours permanent, ces collections devaient donc faire l'objet d'un programme de restauration. Celui-ci a été engagé dès l'année 2001 et devra se poursuivre selon un calendrier défini par ailleurs, d'abord jusquà la réouverture puis en fonction des programmations d'expositions. Ainsi, près de 1800 montages taxidermiques ont d'ores et déjà été restaurés. Les futures présentations permanentes (et semi-permanentes) reposeront numériquement et principalement sur ces collections patrimoniales. Néanmoins, de nouvelles collections sont à créer pour plusieurs raisons.

\section{Le renouvellement de matériaux périssables}

Les collections d'animaux naturalisés sont composées de matériaux organiques, périssables à plus ou moins long terme, qui n'ont pas toujours été conservées dans les meilleures conditions dans le passé ; il importe donc de les renouveler régulièrement. En particulier pour la présentation au public des espèces régionales, il peut être plus pertinent de créer une nouvelle taxidermie à partir d'une dépouille récemment collectée et bien documentée que de restaurer un spécimen ancien qui conservera néanmoins un intérêt scientifique de référence.

\section{L'exhaustivité ciblée}

Les musées visent à une certaine exhaustivité, définie dans ce qu'on appelle aujourd'hui leur projet scientifique et culturel (PSC). Il est indispensable de compléter les familles taxonomiques ou les ensembles environnementaux que nos prédécesseurs ont commencé de constituer. Dans le cadre du projet de rénovation, les priorités à l'intérieur de ce domaine entrant dans nos missions fondamentales, ont été détaillées dans le projet muséographique défini par l'équipe scientifique.

\section{L'adéquation au projet} scientifique et muséographique

- des spécimens plus attractifs

Le projet scientifique développe un certain nombre de thématiques environnementales qui seront mises en espace

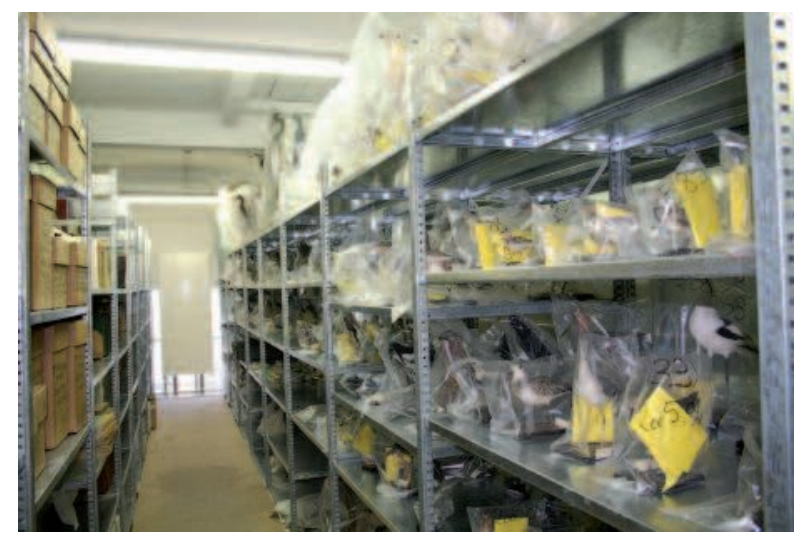

Le local de réserves situé au centre ville, à proximité du muséum, a permis l'organisation rationnelle des collections non exposées et leur accessibilité. () Muséum de Bordeaux

grâce à une scénographie adaptée. Pour ce faire, les collections patrimoniales seront bien évidemment utilisées en priorité. Néanmoins, le propos de nos prédécesseurs était principalement focalisé sur la classification et les collections recueillies étaient destinées à illustrer ce propos. Ainsi, par exemple, les collections de minéralogie et de géologie comportent des séries d'échantillons de taille modeste, permettant l'exposition du plus grand nombre de variétés. Aujourd'hui, on frappera davantage l'esprit du public avec des spécimens moins nombreux mais de dimensions et de qualité supérieures. De même, les montages taxidermiques sont réalisés dans des postures standard très neutres, permettant la comparaison des espèces. Pour l'illustration de propos comme la locomotion, la prédation, la reproduction, l'élevage des petits... nous nous attachons à compléter les collections par des montages en postures dynamiques diversifiées, créés « sur mesure ».

- des créations permettant de voir ce qui est caché

L'un de nos prédécesseurs au début du XXe siècle, le professeur J. Kunstler, l'avait bien compris : d'autres types de collections sont également indispensables à ce qui ne s'appelait pas encore la médiation scientifique. Des moulages, des modèles à échelles diverses, des écorchés (montrant l'anatomie interne des organismes), permettent en effet d'illustrer un propos autre que le propos de classification. Les collections qu'il a constituées à l'époque sont précieuses ; il nous faut les compléter car ses successeurs n'ont pas prolongé les acquisitions dans cette direction, indispensable pour expliciter les aspects biologiques et physiologiques de notre propos et prendre en compte les connaissances scientifiques actuelles. Car, pas plus que J. Kunstler en 1905, nous n'avons pour ambition de nous limiter à refaire le musée du XIXe siècle.

\section{Des collections aux usages divers induisant des statuts différenciés}

Le muséum d'Histoire naturelle de Bordeaux, comme l'ensemble des muséums de France, est sous la tutelle 
scientifique du ministère de l'Enseignement supérieur et de la Recherche (ou, dans le passé du ministère de l'Éducation nationale) et non sous celle du ministère de la Culture. De ce fait, et du fait de la particularité de leurs collections considérées principalement comme objets d'enseignement et de recherche, échantillons représentatifs renouvelables et non objets patrimoniaux, l'inventaire des collections n'était pas réalisé selon les règles s'appliquant aux œuvres d'art.

Néanmoins, la loi sur les Musées de France de janvier 2002, suivie de l'arrêté de mai 2004, a souligné l'importance de l'inventaire des collections. L'obligation d'un récolement décennal pour les établissements relevant de celleci a été spécifiée dans la circulaire du 27 juillet 2006. Le fait, pour les muséums, d'avoir sollicité et obtenu l'appellation « Musées de France », les oblige à un tri dans leurs collections et à une ventilation de celles-ci selon le statut qu'il semble le plus opportun de leur attribuer.

\section{Les collections patrimoniales} ou collections «Musées de France »

Certains spécimens représentent effectivement ce que l'on peut rapporter à des collections patrimoniales ou collections au sens des «Musées de France ». Ces collections sont non renouvelables pour une part de plus en plus importante ; il s'agit en effet d'individus d'espèces menacées et protégées ou parfois même récemment disparues, ou de fossiles ou minéraux qui ont été largement exploités ou ne sont plus accessibles aujourd'hui. Elles peuvent être exposées de façon permanente ou temporaire, ou être conservées en réserve et faire l'objet de consultation par des chercheurs ou des étudiants dans la discipline considérée.

Le statut de protection de ces collections «Musées de France » est le plus élevé, réglementé par les dispositions du Code du Patrimoine (2004) qui a suivi la loi sur les Musées de 2002 et refondait les dispositions de 1945. Ces dispositions sont communes à l'ensemble des collections des Musées de France (art, histoire ou sciences et techniques). Les objets ou spécimens auxquels ce statut est conféré deviennent inaliénables, c'est-à-dire qu’ils ne peuvent être vendus ni échangés. Par ailleurs, ce statut entraîne pour le musée l'obligation de maintenir ces objets ou spécimens dans les meilleures conditions de conservation.

Afin que la mise en œuvre de ces missions puisse être contrôlée, les collections concernées sont soumises à un inventaire réglementaire réalisé suivant des normes très précises et, depuis 2004, à l'obligation de procéder tous les dix ans à un récolement (c'est-à-dire le rapprochement de cet inventaire écrit du constat de présence et d'état des objets ou spécimens dans les vitrines des salles d'exposition ou sur les rayonnages des réserves). Le récolement actuellement en cours doit donc être achevé en 2014.

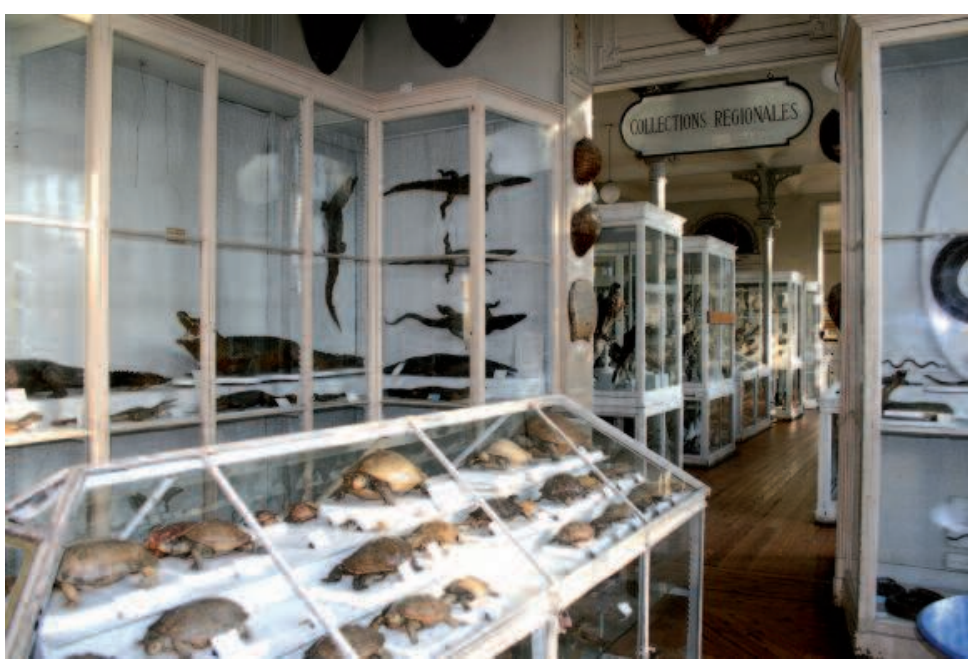

Les salles permanentes du premier étage : sur le palier, la collection générale des reptiles et amphibiens, et perspective vers la salle des collections régionales (c) Muséum de Bordeaux

Toute intervention de restauration conduit à une modification de l'objet. Depuis 2002, toute restauration de ces collections est soumise à l'avis d'une commission scientifique régionale composée de pairs, qui statue sur l'opportunité de cette restauration mais aussi sur le respect d'un protocole à suivre, les matériaux à utiliser ainsi que l'adéquation des compétences du restaurateur avec la nature des collections et la hauteur des dégradations.

Il convient donc d'être vigilant et de clairement définir les critères d'entrée en collection en raison des responsabilités et des conséquences en termes de moyens humains et financiers nécessaires que ce statut impose. C'est pourquoi une autre commission, composée de façon équivalente, statue sur les acquisitions, autrement dit sur l'opportunité de faire entrer un nouvel objet dans les collections « Musées de France ». Parmi les critères de réception au sein de ces collections, la précision et la richesse de la documentation qui accompagne le spécimen sont un facteur déterminant.

\section{Les collections d'étude}

Depuis leur création, les muséums ont acquis des collections à des fins d'études et de recherche, ces dernières pouvant être partiellement destructrices ; ces collections ne peuvent donc pas s'inscrire dans la même logique patrimoniale que les précédentes.

Les collections d'étude doivent faire l'objet d'inventaires, souvent par lots plus larges, distincts de l'inventaire réglementaire des collections « Musées de France ». Le prélèvement d'une partie pour analyse ou même la destruction d'un échantillon complet pour analyse fait partie intégrante de la destination possible de ces collections.

Mais le statut de collection d'étude constitue souvent un «sas » et c'est l'étude qui indiquera s'il est pertinent de 
proposer le statut patrimonial à tout ou partie de la collection qui peut devenir alors une référence pour la communauté scientifique internationale.

\section{Les collections pédagogiques}

Enfin, la vocation d'enseignement des muséums est inscrite dès l'origine de leur création ainsi qu'en témoigne la tutelle scientifique du ministère de l'Éducation nationale (ou de celui de l'Enseignement supérieur et de la Recherche aujourd'hui).

Certaines séries sont acquises pour leur intérêt pédagogique. Elles sont composées de spécimens souvent démonstratifs, mais renouvelables (moulages, maquettes, séries aisément rééchantillonnables), ou parfois de séries non documentées si elles peuvent néanmoins permettre une activité nécessitant des manipulations par le public. Dans ce cas, on considère leur usure et leur renouvellement comme s'inscrivant normalement dans leur statut.

\section{Les dépouilles en attente}

Les collections de spécimens naturalisés et les collections d'ostéologie sont créées par des taxidermistes ou des préparateurs-monteurs, prestataires ou salariés des musées, à partir de dépouilles collectées auprès d'organismes habilités ou parfois de particuliers. Ces dépouilles, dont l'arrivée est aléatoire, sont stockées en congélateurs, en attente d'un projet muséographique : certaines seront en effet tannées et éventuellement montées ou utilisées pour les pièces osseuses, séparées ou en montage. Une dépouille fournira alors plusieurs spécimens de collections qui entreront éventuellement en collections patrimoniales. Parfois, elles ne pourront être traitées avec succès et seront détruites. D'autres encore pourront être proposées à d'autres musées.

Les dépouilles en attente font l'objet d'un tableau d'entrée séparé et d'une numérotation provisoire puisqu'il s'agit

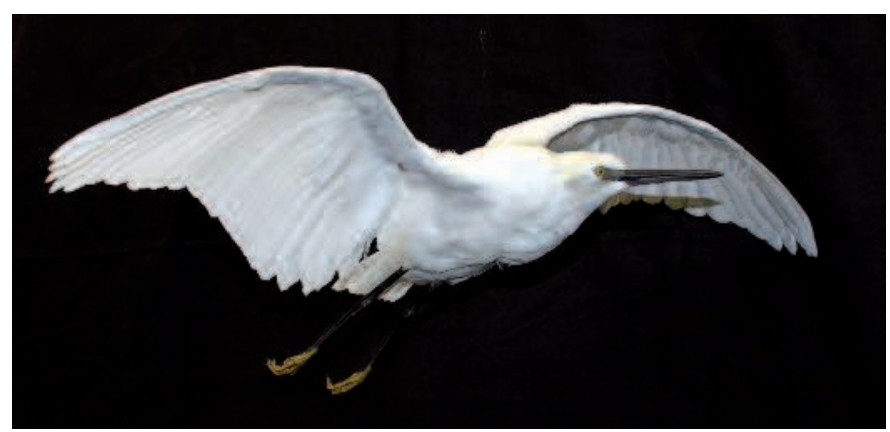

Aigrette garzette en vol : montage taxidermique réalisé par Yves Walter en 2009, à partir d'une dépouille confiée par le centre de soins d'Audenge (Ligue pour la Protection des Oiseaux), à destination de la première exposition de l'espace régional du futur parcours semi-permanent Le littoral aquitain. (c) Muséum de Bordeaux

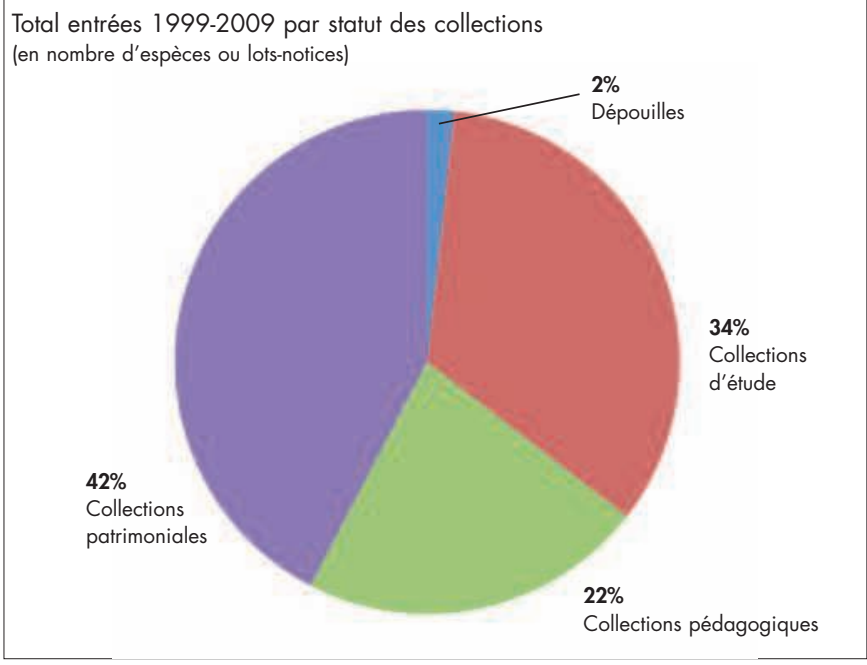

Les acquisitions par types de collections

toujours d'un état transitoire. Au fur et à mesure de l'affectation des dépouilles pour constituer un ou plusieurs «objets » de collections, ces listes sont actualisées et modifiées : le devenir de chaque dépouille (y compris parfois la destruction) est précisément consigné car la traçabilité entre la dépouille et le ou les spécimens préparés à partir de celle-ci doit pouvoir être suivie.

Dans tous les cas, la réglementation sur les espèces protégées s'applique. Seuls les établissements et les professionnels habilités sont autorisés à transporter, à traiter puis à exposer les spécimens correspondants.

\section{Différents modes d'acquisition}

C'est donc avec la perspective de la rénovation de l'établissement et, en 2001, l'aménagement d’un local de réserve adapté, que cette nouvelle politique d'acquisition a pu être définie et progressivement mise en place. En dix ans, près d'une centaine d'entrées ont été enregistrées et correspondent à environ 18000 spécimens. Principalement issues de dons, ces acquisitions ont aussi été le fait d'achats, d'un legs et de dépôts.

\section{Les dons}

Les dons concernent des collections de nature, d'ampleur et de niveau d'intérêt très divers. Les séries entrent, dans un premier temps du moins, en collection pour étude, l'étude permettant d'en déterminer l'attribution finale.

Les dépouilles, conservées en congélateurs, restent en attente jusqu'à ce qu'elles fassent l'objet d'un projet de taxidermie ou de préparation ostéologique pour leur intégration en collections pédagogiques ou patrimoniales.

La nouvelle politique d'acquisitions a conduit, d'une part à être plus sélectif dans l'acceptation des dons spontanés et à préciser au donateur la destination qui en serait faite, et d'autre part à venir au-devant des donateurs et à solliciter 


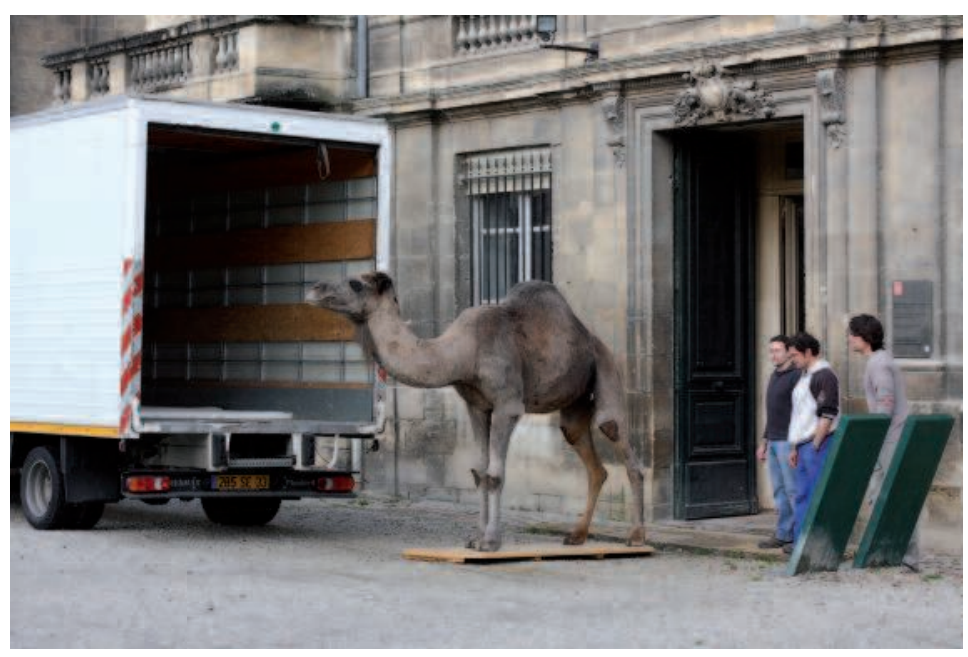

Dromadaire naturalisé par Philippe Engel, taxidermiste. La dépouille a été récupérée par le prestataire au zoo de Thoiry en 2008. Le dossier a été soumis à la commission d'acquisition en 2009 et a bénéficié d'une subvention du FRAM ; il a été livré au muséum en mars 2011. Il figurera parmi les spécimens phares du futur parcours permanent décliné autour de la thématique générale La nature vue par les hommes. (c) Muséum de Bordeaux

des dons sur projet (avec des particuliers) ou en établissant des conventions avec des organismes habilités (en particulier pour la collecte de dépouilles en vue de préparation de collections).

Les dons représentent néanmoins $77 \%$ des entrées depuis dix ans, et $65 \%$ des spécimens ; ils correspondent à quarantaine de donateurs différents.

\section{Les dons spontanés}

Les dons spontanés représentent environ 1/3 des entrées réalisées. Les donateurs ont deux profils : il peut s'agir directement des collecteurs ou collectionneurs mais aussi souvent des descendants de ceux-ci ou de leur conjoint veuf ou veuve. Dans le premier cas, le spécimen ou la série sont généralement documentés (mais pas toujours), dans le second, la documentation est le plus souvent inexistante ou incomplète.

Toutes les propositions de dons ne peuvent donc pas être acceptées, ainsi :

- des objets ou spécimens n'entrant pas dans le projet scientifique de l'établissement,

- des spécimens en mauvais état sanitaire,

- des spécimens d'espèces protégées par la Convention de Washington (CITES : Convention of International Trade of Endangered species, Convention sur le commerce international des espèces sauvages menacées d'extinction) collectés après 1973 et proposés par des personnes non habilitées.

Les spécimens peu ou mal documentés peuvent néanmoins être acceptés si leur qualité représentative ou esthétique permet de les utiliser pour des animations ou ateliers, parfois même pour des expositions (selon le propos qu'ils pourront illustrer) ; ils entrent alors en collections pédagogiques.

\section{Les dons sur projets}

- une pratique ancienne : dès l'origine du cabinet d'Histoire naturelle qui prendra le nom de muséum en 1862, les conservateurs en charge des collections ont sollicité des correspondants qui recherchaient, pour le compte de l'établissement, des spécimens et échantillons susceptibles d'enrichir les collections. En raison des différentes législations de protection des espèces et des sites, cette politique ne peut aujourd'hui être menée que de manière limitée avec des particuliers, de manière plus large avec les organismes habilités.

- les particuliers avec lesquels des dons sur projet ont été menés au cours de la dernière décennie sont des membres de sociétés savantes, amateurs ou chercheurs professionnels (universitaires), qui ont réuni et documenté des spécimens ou échantillons dans l'objectif de les intégrer aux collections du musée après accord informel avec le conservateur. - avec les organismes habilités (Office national de la chasse et de la faune sauvage, parcs nationaux, certaines associations, zoos, autres muséums), des conventions ont été établies afin que le muséum puisse être destinataire de dépouilles d'espèces (éventuellement protégées) qui auraient été destinées à l'équarrissage. Le muséum sélectionne alors parmi les dépouilles disponibles celles qui lui sont nécessaires en vue du projet de rénovation de l'établissement, en vue d'expositions temporaires à venir ou encore en vue d'échanges avec des collègues d'autres muséums. Si on inclut les entrées de dépouilles parmi les opération réalisées, les dons issus de ces organismes représentent $45 \%$ des opérations.

- les collectes directes par le personnel : outre celle réalisée par les correspondants, une part de la collecte d'échantillons et spécimens peut être réalisée directement par le personnel des muséums. C'était le cas par le passé, ainsi qu'en témoignent les permis de chasse « en toute saison » délivrés aux préparateurs et figurant dans nos archives. C'est toujours le cas dans les muséums disposant de grosses équipes qui mènent des activités de recherche sur le terrain (fouilles paléontologiques programmées, missions océanographiques...). Pour le muséum de Bordeaux, ces collectes sont aujourd'hui assez occasionnelles, destinées à compléter des séries pédagogiques (thanatocénoses littorales, échantillons pétrographiques), ou d'opportunité avec la récolte de dépouilles rencontrées de façon fortuite.

\section{Les legs}

Le legs est la transmission d'un ou plusieurs biens du défunt, faite par testament de son vivant, mais qui ne prendra effet qu’à son décès. 
Entrée : novembre 2004

\section{Fonds d'atelier de taxidermie}

Collecteur : François Chanudet

Vendeur et donateur :

M. et Mine Claude et Belly Charron

Cette collection a été proposée au muséum de Bordeaux par l'intermédiaire d'Allain Bougrain-Dubourg, président de la Ligue pour la Protection des Oiseaux (LPO), ami de François Chanudet (1920-2003), taxidermiste. F. Chanudet fut dessinateur au Muséum national d'Histoire naturelle puis taxidermiste au muséum de La Rochelle où il assura les fonctions d'assistant du docteur Loppé puis du professeur Duguy jusquà sa retraite en 1983.

Une expertise de cette collection a été réalisée en mars 2004 par Philippe Jourde (LPO) puis, en juillet, une équipe du muséum de Bordeaux s'est rendue sur place pour consultation directe. La collection s'est en effet avérée d'un grand intérêt : documentée de façon extrêmement précise (date de collecte, localisation, sexage, mensurations, âge...) et les montages taxidermiques d'une grande qualité et en parfait état de conservation.

En octobre, la Ville de Bordeaux fit une offre - pour le compte du muséum - pour l'acquisition de ceux des spécimens qui pouvaient être vendus ; il a été proposé (et accepté par les vendeurs) que les espèces protégées fassent l'objet d’un don complémentaire. L'acquisition a bénéficié de la participation financière du FRAM (Fonds Régional d’Acquisition pour les Musées, associant l'État et la Région).

L'achat comprend : 768 pièces (dont 62 spécimens naturalisés, 134 peaux, 550 pièces ostéologiques) ; le don : 186 pièces (dont 26 spécimens naturalisés, 51 peaux, 102 pièces ostéologiques) et 21 boîtes d’insectes. Au total, 73 espèces sont européennes et 16 exotiques. Les Visons (d'Europe et d'Amérique) et le Chevreuil sont les espèces les mieux représentées.

La collection a été inventoriée en 2005 par Yann Ruter, collaborateur scientifique occasionnel au muséum. Les spécimens ont été numérotés et photographiés en vue de l'inventaire informatisé. La saisie des données et l'insertion des photographies dans la base ont été réalisées en suivant. L’opération s'est achevée le 16 juin 2005, soit 7 mois et demi après l'arrivée de la collection. Seul l'inventaire détaillé des boîtes d’insectes reste à réaliser. De la documentation générale a également été donnée par la famille ; elle a été inventoriée par Laetitia Charron, petite-fille des donateurs, lors d'un mois de stage au muséum dans le cadre de sa formation universitaire. Nombre de spécimens ont déjà été utilisés pour des expositions temporaires. La collection, comprenant beaucoup de jeunes individus, a été largement sollicitée pour l'exposition Tous les bébés (mars 2006-janvier 2007). Les Mustélidés - et en particulier les visons d'Europe non montés (peaux tannées) - sont régulièrement empruntés pour des stages de formation des agents de l'ONCFS.

Certaines taxidermies inachevées ont été confiées au taxidermiste-restaurateur Yves Walter pour finitions. Un squelette non monté de rhinocéros a été confié à M. Cornette (société Jeco) pour parachèvement du dégraissage et montage en connexion anatomique.

Inventaire informatisé :

novembre 2004 à juin 2005

MHNBx $\mathbf{N}^{\circ} 2005.2936$ à 2005.3761

Une seule petite collection est entrée de cette manière au muséum de Bordeaux au cours de la dernière décennie et représente $2 \%$ des spécimens.

\section{Les achats}

À partir de 2002, des achats ont été proposés à la Ville de Bordeaux, dans la perspective du renouvellement des collections en vue du projet de rénovation de l'établissement et à l'occasion des expositions temporaires. Dans ce cadre, le muséum a pu profiter de plusieurs opportunités, en participant à des ventes publiques (six fois dans la décennie écoulée) ou en se portant acquéreur d'une collection particulière très intéressante, comme lillustre l'exemple de la fiche descriptive ci-dessus. De façon plus précise, un plan d'acquisition réparti sur 5 ans, prenant en compte chacun des thèmes du projet scientifique et muséographique, a été proposé à partir de mars 2007. Il porte sur des montages taxidermiques à réaliser à partir de dépouilles en attente, l'acquisition de spécimens, de moulages, modèles ou maquettes permettant d'illustrer certaines thématiques du parcours. Des acquisitions programmées de ce type ont également été réalisées dans le cadre d'expositions temporaires. 


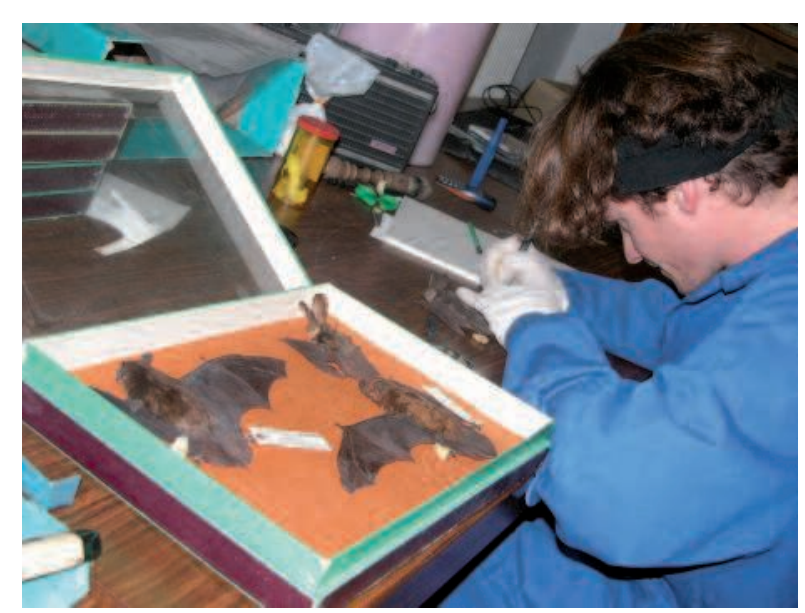

La collection Chanudet en cours d'inventaire et de marquage, à sa réception. (c) Muséum de Bordeaux

Dans le cas des ventes aux enchères les collectionneurs sont parfois connus et les collections documentées, parfois non.

Dans le cas des commandes de moulages ou de montages taxidermiques ou ostéologiques, les prestataires sont des professionnels, taxidermistes ou mouleurs.

De 2002 à 2009, 19 entrées de collections correspondent à des achats de la Ville de Bordeaux pour le compte du muséum, soit $20 \%$ des opérations mais seulement $5 \%$ en nombre de spécimens, ceux-ci ayant souvent été des pièces uniques et plus rarement des séries.

Certaines opérations ont pu bénéficier de subventions du FRAM (Fonds Régional d'Acquisition pour les Musées) financé par l'État (ministère de la Culture) et la Région. Huit dossiers ont été présentés et ont ainsi bénéficié d'une subvention ; quatre de ces opérations figurent sur le site internet du FRAM Aquitaine ; de nouveaux dossiers,

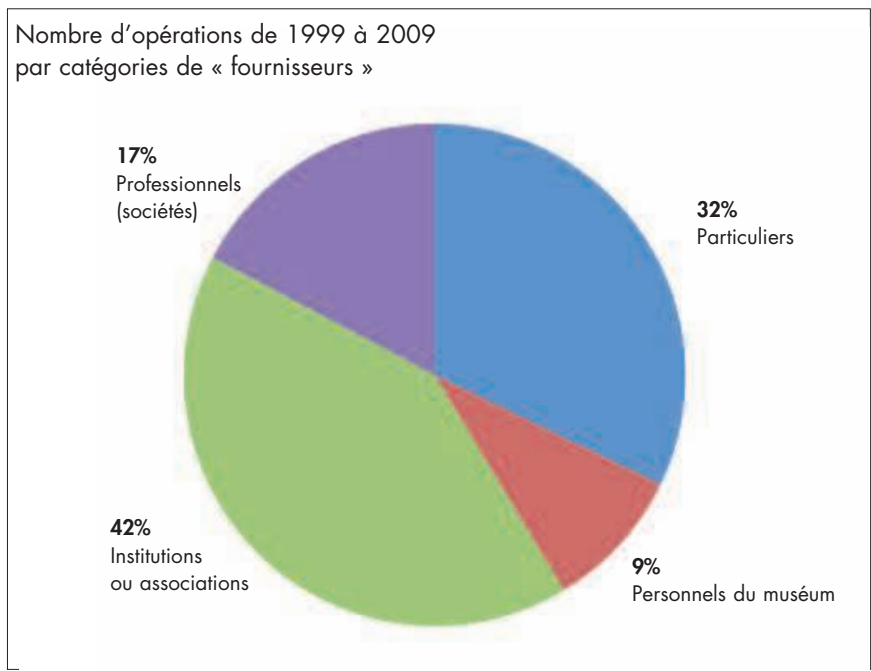

Les différents « fournisseurs » du muséum dont les réalisations sont en cours ou viennent juste d'être livrées, ont été acceptés en 2009 et 2010.

\section{Les dépôts}

Il s'agit d'objets ou de collections confiés par un établissement à un autre qui en a la garde pour une durée définie dans le cadre d'une convention et selon des modalités définies par cette convention. Ces objets ou spécimens font l'objet d'un inventaire particulier, dont la numérotation est réglementairement précédée de la lettre D dans le catalogue de l'établissement dépositaire. Celui-ci ne peut intervenir sur l'objet (restauration, prêt...) sans l'accord du déposant. Une collection de minéralogie de l'université Bordeaux 1 a ainsi été déposée au muséum en 2003 (voir l'exemple de la fiche descriptive ci-contre).

\section{La présentation au public}

Les nouvelles acquisitions sont régulièrement présentées au public. Jusqu'à la fermeture de l'établissement, certaines étaient, dans la mesure du possible, insérées au parcours permanent, d'autres réalisées à l'occasion d'expositions temporaires ont été exposées aussitôt. En janvier 2010, une exposition récapitulative hors les murs, intitulée 10 ans d'acquisitions, a permis de présenter durant 10 jours un échantillonnage de celles-ci, à la fois représentatif des objectifs, de la nature des spécimens, du mode d'entrée, ce qui a permis de rendre hommage aux donateurs et de mettre en valeur le travail des prestataires, taxidermistes et sculpteurs-mouleurs.

\section{Conclusion}

Très active entre la deuxième moitié du XIXe siècle et le premier tiers du XXe siècle, la politique d'acquisition du muséum de Bordeaux s'est ensuite limitée à des entrées de dons spontanés de particuliers, les derniers achats, très ponctuels, remontant à 1975 .

La décennie 1980-1990 a été consacrée au sauvetage des collections mises en péril par une importante inondation (1982) et au lancement des premières expositions temporaires permettant de renouveler l'intérêt pour les thématiques naturalistes. La décennie suivante a permis de développer une véritable politique d'expositions et de médiation scientifique (animations scolaires et familiales, conférences, animations théâtrales...) autour du fonds des collections qu'il s'agissait de faire redécouvrir et de valoriser, en présentant des sélections ciblées autour de thématiques variées. Cette orientation a permis de doubler et même tripler la fréquentation de l'établissement.

Une nouvelle politique d'acquisitions n'a véritablement été engagée qu'avec le lancement de la réflexion sur la rénovation de l'établissement menée en parallèle du chantier 
Entrée : octobre 2003

\title{
Collection Robert Gay : minéralogie générale et minéraux des Pyrénées
}

\author{
Collecteur : M. Bonnal \\ et laboratoire de cristallographie UBx1 (Pr. Robert Gay)
}

Déposant :

Université Bordeaux 1

Une convention passée en septembre 2003 entre l'université de Bordeaux 1 et la Mairie de Bordeaux a permis de mettre en dépôt au muséum d'Histoire naturelle la collection de minéraux de l'ancien laboratoire de cristallographie.

Vingt-quatre blocs-meubles de 10 tiroirs chacun ont été transférés. La couverture photographique de chaque tiroir a été réalisée en juillet 2003, avant le transfert en octobre.

La collection de minéraux des Pyrénées ou collection Bonnal a été achetée par le laboratoire en 1960. Avec 1000 entrées au fichier, elle n'a sans doute pas d'équivalent en France. La collection générale (reste du monde) compte 4000 entrées; elle résulte de la fusion des collections initiales du laboratoire de cristallographie et de la partie étrangère de la collection Bonnal que ce dernier avait constituée par échanges.

À l'occasion du dépôt, il a été convenu que cette collection porterait le nom de « collection Robert Gay » en hommage à celui qui fut le principal artisan de sa constitution. C'est Marc Alléaume, ancien enseignant-chercheur de ce laboratoire, qui a été à l'initiative du dépôt de la collection au muséum. En mars 2004, une cérémonie officielle de réception de la collection a été organisée au muséum, en présence des différents partenaires et de Hubert Curien, ancien ministre de la Recherche, à l'initiative de la Fête de la Science.

La collection est généralement bien documentée par ses étiquettes de détermination et provenance ; les échantillons sont le plus souvent numérotés avec renvoi à un fichier papier qui distingue trois collections (indiscernables par leur numéro seul) :

- la collection générale, notée CG ;

- la collection régionale, notée CR, qui comprend une très grande partie de spécimens échantillonnés dans les Pyrénées ;

- la collection de travaux pratiques, notée TP.

La collection a été récolée et inventoriée sur la base de données informatisée des collections du muséum. La couverture photographique par échantillon a été réalisée pour ceux des spécimens pré-sélectionnés en vue d’exposition dans le futur parcours muséographique. Le total a permis de recenser précisément 5221 échantillons. Ce travail, réalisé en 2008 et en 2009 par Marie Jullion, géologue, formée à l'inventaire des séries de minéralogie sur la collection de Jussieu (Paris 6), a représenté 7 mois d'équivalent temps plein.

Inventaire informatisé :

2008-2009

MHNBx D.2008.CG.112.1 à D.2009.TP.95.131.2

des collections. Aujourd'hui l'inventaire rétrospectif informatisé des collections qui étaient exposées dans les salles permanentes est achevé, les restaurations envisagées pour la réouverture avancées à plus de $80 \%$, les achats envisagés sont réalisés également à près de $80 \%$. Restent les acquisitions d'opportunités (propositions d'achats ou dons) qui continueront d'enrichir le projet et les futures expositions. Par la suite, deux objectifs seront poursuivis : celui du renouvellement des séries anciennes, dans la mesure du possible, et celui de l'enrichissement des séries existantes qui font la spécialité de l'établissement. 